\title{
Ebola hemorrhagic fever
}

INSERM

\section{Source}

INSERM. (1999). Orphanet: an online rare disease and orphan drug data base. Ebola hemorrhagic fever. ORPHA:319218

Ebola hemorrhagic fever (EHF), caused by Ebola virus, is a severe viral hemorrhagic disease characterized by initial fever and malaise followed by gastrointestinal symptoms, bleeding, shock, and multi-organ system failure. 